\title{
HUMAN ACTIVITY RECOGNITION USING ACCELEROMETER DATA WITH MULTI CLASS SVM
}

\author{
Anju M Nair \\ Department of CSE \\ Rajiv Gandhi Institute of Technology, \\ Kottayam, Kerala, India
}

\begin{abstract}
Human Activity Recognition is a process of classify different human activities in their daily livings. Various human activity recognition techniques are proposed in some research studies. Human activity recognition is important in health-care monitoring systems for monitoring abnormal behavior of patients. Previous methods are based on using multiple sensors but it leads to obstructive movements. In the proposed system, which uses a single sensor data called accelerometer data. Both time-domain features such as mean, deviation, etc and frequency-domain features such as signal entropy, kurtosis, etc are extracted. Dimensionality reduction is applied using both ICA and PCA. Compare both ICAbased and PCA-based features. Classification using Multi class SVM gives $95.51 \%$. Individual activity performance is also measured.
\end{abstract}

Keywords-Accelerometer, Machine learning algorithms, Activity classification

\section{INTRODUCTION}

Human Activity Recognition (HAR) have a great significance in computer vision. Human activity recognition is a growing area in many domains mainly in the case of Health Care Monitoring (HCM) applications, where abnormal behaviour of patients can be monitored by sensing repeated activity movements [17]-[19]. In surveillance, cameras installed in the areas might needed monitoring such as banks, airports, etc. Currently, surveillance systems are mainly focusing on recording. The aim of activity detection using Closed-circuit television (CCTV) is to detect mistrustful activities like violation and thieve. In some applications like location-based services and a numerous systems, that involve inter-linkage between human and electronic devices, human activity recognition plays an important role. Human activities can be recognized from videos, images and also using sensors from our mobile like accelerometer, gyroscope, etc.

Accelerometer is a sensor used in smartphone that measures linear and angular acceleration. Accelerometer device measures proper acceleration in every seconds. Accelerometer consists of three axes, $\mathrm{X}, \mathrm{Y}$ and $\mathrm{Z}$. X measures horizontal movements of the body. Y-axis is for the vertical movements only. In addition, if the device face up on a table, the Z-axis measures acceleration of earth gravity and is 9.81 $\mathrm{m} / \mathrm{s} 2$. The $\mathrm{X}$ and $\mathrm{Y}$ axes, which are perpendicular to the acceleration of earth gravity, both output $0.0 \mathrm{~m} / \mathrm{s} 2$. Another type of sensor is gyroscope, measure orientation. The main drawback of existing system is that uses multiple sensors for the activity recognition, which leads to obstructive movements and settings are not feasible in practice [23]. In the proposed system, this uses only a single sensor data called accelerometer data. For human activity recognition, accelerometer data is enough to recognize activity.

Human activity recognition is to find the activities of daily livings (ADLs). Mainly, there are two types of human activities: Locomotive human activities and Semantic human activities [26], [27]. Locomotive human activity is the activity that is doing in the daily life examples jumping, standing, walking, sitting, stair up etc. Semantic human activities are activities like shopping, having dinner, viewing videos, etc. But proposed system consider only locomotive activities jumping, sitting on a chair, standing, stair up, stair down and walking.

Area of research in human activity recognition is based on machine learning methods. Machine learning is an application of artificial intelligence. Proposed system is about Human activity recognition using machine learning techniques. Machine learning is used to make best performance standard using examples or past experience. Learning is used when doesn't exist human expertise, humans are unable to explain their expertise, solution needs to be adapted to particular cases and solution changes in time, etc. Machine learning is the study of a process or set of rules to be followed in calculations or other problem-solving operations, especially by a computer, at some task with experience which improve their performance. For speech recognition, computer vision, natural language processing, medical outcomes analysis, robot control, computational biology, etc., machine learning is the preferred approach. This trend is accelerating improved machine learning algorithms, networking, improved data capture, faster computers, new sensors/IO devices, demand for self-customization to user environment, software too complex to write by hand. It turns out to be much effort to extract 
knowledge from human experts because failure of expert systems in the 1980s [15]-[16].

Learning is any process by which a system make better performance from practical contact. Machine learning is concerned with computer programs that automatically improve their performance through observations. Machine learning develop systems that can automatically modify and customize themselves to individual users, example: filtering mails. It discovers knowledge from database. Machine learning have an ability to imitate human and replace certain repetitious tasks, which require some intelligence like identifying handwritten characters. Machine learning applications are : Image processing, Bioinformatics, Natural language processing, Computer vision and Marketing.

The input to a learning algorithm is training data, representing observations, and the output is any procedure, which usually takes the form of another algorithm that can perform a piece of work. The input data to a machine learning system can be numerical, multimedia textual, audio, or visual. The corresponding output data of the system can be an integer representing a category, for instance a class, for example, a pigeon or a sunflower from image recognition or a floatingpoint number, for instance, the velocity of a rocket.

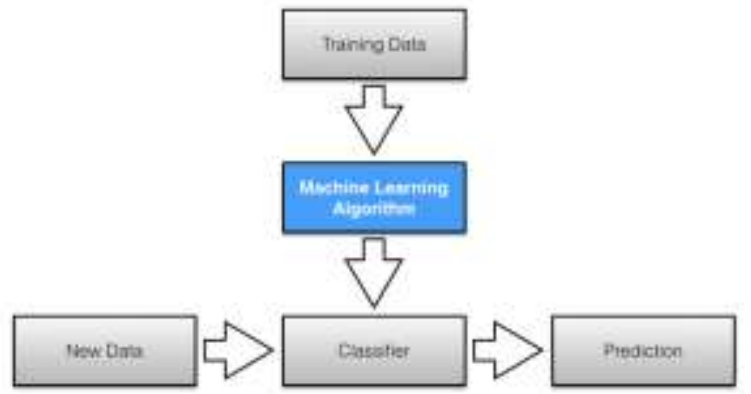

Fig. 1. Basics of Machine learning algorithms

In all machine learning techniques, first step is data acquisition. Data acquisition is a process of collection data from different sources for prediction. So, it should consist of sufficient features for prediction. This collected data are treated as training data. Then develop a model using machine learning algorithms like supervised learning algorithms, unsupervised learning etc. Then put this into a classifier like SVM. Classifier take test data as input and output is the predicated class.

\section{A. Supervised Learning}

In supervised learning, learning data comes with explanation, labels, desired outputs and the objective is to find a common rule that maps inputs to outputs. This kind of learning data is called labelled data. To label new data with unknown output, a learning rule is applied. Supervised learning build a model that is based on labelled samples. For example, if build a system to classify human activities such as jumping, sitting, standing, etc, first need to create a database and label it. Based on this data, the algorithm will learn how to classify activities of human using values of input features. From the available data, supervised learning deals with learn a function. Some supervised learning algorithms are Logistic Regression, Support Vector Machines (SVMs), Neural networks, and Naive Bayes classifiers. Classifying e-mails into spam and not-spam categories, labelling web pages based on their content, and voice recognition are some examples of supervised leaning.

\section{$B$. Semi-supervised Learning}

If some learning samples are labelled, but some others are not labelled, then it is semi-supervised learning. It makes use of a large amount of unlabelled data for training and a small amount of labelled data for testing. Semi-supervised learning is applied in cases where it is expensive to acquire a fully labelled dataset while more practical to label a small subset. For example, it often requires experienced experts to label many field experiments to locate oil at a particular location and certain remote sensing images, while acquiring unlabelled data are relatively easy.

\section{Unsupervised Learning}

To detect irregularity, outliers, such as wrongful or defective equipment, or to group customers with similar behaviours for a sales campaign, unsupervised learning is used. It is completely different from supervised learning. There is no labelled data here. it is the duty of coder or algorithm to find the composition of the data, When learning data contains only some indications without any description or labels. This kind of learning data is called unlabelled data. So, an unsupervised learning algorithm seeks to classify the given dataset into a certain number of groups optimum.

In the proposed system, HAR is performed using a single sensor that is accelerometer data. The dataset is publicly available [14]. A dimensionality reduction technique applied after feature selection and extraction. For dimensionality reduction, Principal component analysis (PCA) and Independent Component analysis (ICA) are used. Compare both PCA and ICA result with classifiers. Naive Bayes (NB) and Multi class Support Vector Machine (SVM) machine learning classifiers used for classification. PCA-based features and ICA-based features compared with Naive Bayes and multiclass SVM.

The scopes of the proposed system are: Human activity recognition is important in health care monitoring application where monitoring abnormality of patients. In addition, activity recognition is research oriented in the area of computer vision and biomedical engineering. In addition, the objectives are:

- Introduce two methods for feature reduction (dimension reduction), PCA and ICA.

- Compare PCA and ICA with classifiers Naive Bayes and multiclass SVM. 


\section{International Journal of Engineering Applied Sciences and Technology, 2019 \\ Vol. 4, Issue 3, ISSN No. 2455-2143, Pages 374-381 \\ Published Online July 2019 in IJEAST (http://www.ijeast.com)}

Previous studies about human activity recognition uses multiple sensor data like accelerometer, gyroscope, orientation, etc. However, the use of multiple sensors leads to obstructive movements and the settings are not feasible in practice. Accelerometer data itself is enough to classify human activities.

\section{PREVIOUS WORK}

More than one HAR approaches have been present in various research areas. Almost all of these studies are composed of two groups of methods. The first one involves the utilization of probabilistic models to deduce the types of activities. The second one uses classification techniques that map inputs of sensory data to the desired class.

Yuxin Peng et al. utilize time series shapelets for human activity recognition in 2015 [6]. For 4 activities, model a multilayered model. For evaluation, basketball play and activity of daily living are considered. By using multiple sensor for activity recognition the cost increases also there is an obstruction in the movement occur. Tam Huynh et al. proposed a method to recognize daily routines as a probabilistic combination of activity patterns in 2014 [7]. The use of topic models enables the automatic discovery of such patterns in users daily routine. To find the activity pattern become insufficient for discovering semantic activities.

Zhixian YAN et al. proposed an evaluation for the ability of locomotive signatures to deduce semantic activities (Human Activity) in realistic environments, using data from a single embedded accelerometer [5] in 2012. Longitudinal activity data are not considered in the human activity recognition. Dipanjan Chakrabort et al. exploit an empirical investigation of energy consumption characteristics of real-time activity recognition applications 2012 [9]. The investigation indicates that such an activity-adaptive model holds potential for significantly reducing the energy overheads of accelerometer based continuous mobile sensing. A continuous activity recognition generate accelerometer data, but classifier cannot differentiate two activities that is one activity depend on another one.

Y. Lee et al. exploit a method to recognize activities using hierarchical probabilistic models 2011 [11]. The system is composed of four components, which are context collection, preprocessing and feature extraction, activity recognition, and an interface for visualization and labelling. Bayesian network models for activity refer to context hierarchy and activity hierarchy. Which need a multi sensor network. That leads to obstruction movement.

Z. Zeng and Q. Ji, a dynamic Bayesian network (DBN) learning with domain knowledge is proposed for Human Activity Recognition 2010 [10]. To guide DBN uses different types of domain knowledge, regarding first order probabilistic logic. Structure priors and parameter constraints are two model priors transformed by FOPLs. A large training dataset is obtained. However, it leads to high computational cost and more time to training.
R. Cilla et al. exploits a method for selecting features for Human Activity Recognition in 2009 [12] from sensors is presented. Features are selected from Best First Search to improve the accuracy of Hidden Markov Model. Multiple sensors are used here for the recognition. It leads to Obstruction movement. Ling Bao et al. Intille proposed algorithms are developed and evaluated to detect physical activities from data acquired using five small bi-axial accelerometers worn concurrently on distinct units of the body in 2004 [8]. The accuracy of detect some common everyday activities are difficult.

Jie Fu et al. proposed a Bayesian conditional probability with latent-structure model for context-aware activities of daily living (ADL) recognition [4]. RGBD sensor (Microsoft Kinect) is used for obtaining input data. Features are selected based on RGBD Sensor signals. By using RGBD sensor both intra and inter activities are recognized. However, data that are more irrelevant grab each second.

Younghwan Yoo et al. propose the possibility of recognizing and predicting user activities in the IoT (Internet of Things) based smart environment [13]. Activity pattern clustering and activity type decision are the two steps of activity recognition that tries to find the best combination of a pattern clustering method. The computational overhead is high in pattern clustering.

\section{PROBLEM STATEMENT}

Human Activity Recognition Using sensor-based data is an important area of research. Here, human activities of daily living (ADL) such as jumping, standing, sitting, stair up, stair down, and walking are recognized using accelerometer sensor data. How Human Activity Recognition (HAR) is done:

- To classify human activities, accelerometer signal based features and dimensionality reduction techniques are used.

- $\quad$ Predict the class of new data using Multi Class

\section{PROPOSED SYSTEM}

Human Activity Recognition Using sensor-based data is an important area of research. Here, human activities of daily living (ADL) such as jumping, standing, sitting, stair up, stair down, and walking are recognized using accelerometer sensor data. The Dataset is publicly available. The raw data are transformed into an understandable format by using data preprocessing. Data cleaning, Scaling and labelling are the techniques performed in data preprocessing step. After data preprocessing, feature selection is done. The features are subjected to Dimensionality reduction. The final step of activity recognition is classification. Machine learning techniques like SVM, Naive Bayes are used for classify activities. 


\section{A. Basic Architecture}

From Figure. 2 shows that the first step is data acquisition. The raw data is publicly available [14] called MobiFall Data set. This data set consist of six activities of daily living (ADLs).

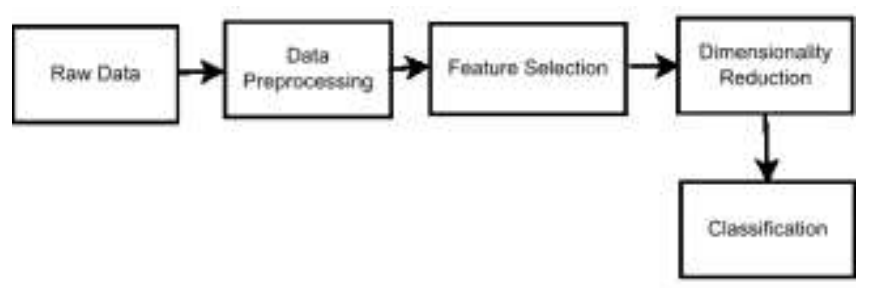

Fig. 2. Proposed Architecture of human activity recognition (HAR)

Data is accelerometer based sensor data. Dataset consist nine person with six activities each. Totally 207 samples are included in the dataset. After data acquisition is completed, the next step is data preprocessing. Data preprocessing consist of the acquired data is subjected to cleaning and labelling. That is, data is transformed into an understandable format. In cleaning unnecessary information, regarding data are removed. Data labelling step is to label each activity as per the ids. In feature selection, accelerometer signal based features like mean, variance, standard deviation, kurtosis, skewness etc are selected. Using PCA and ICA, dimensionality reduction process is done. These reduced features are treated as training set. Then undergoes classification, it predicts the class. For classification Multi class SVM is used.

\section{METHODOLOGY}

For Human Activity Recognition, many studies and researches are already performed. In the proposed system, Data Acquisition, Data preprocessing, feature selection, dimensionality reduction and classification are the steps for activity recognition.

\section{A. Data Acquisition}

Data Acquisition is a process of collecting data from different sources. Here, Dataset is publicly available [14] called Mobifall. In this dataset, data is collected from accelerometer. Accelerometer sensor is used for measuring acceleration or body movements.

TABLE 1. ACTIVITIES OF DAILY LIVINGS (ADLS)

\begin{tabular}{|c|c|c|c|c|}
\hline id & Code & Activity & Trails & Duration \\
\hline 1 & JUM & Jumping & 3 & $30 \mathrm{~s}$ \\
\hline 2 & SCH & Sit on Chair & 6 & $10 \mathrm{~s}$ \\
\hline 3 & STD & Standing & 1 & $5 \mathrm{~m}$ \\
\hline 4 & STN & Stair Down & 6 & $10 \mathrm{~s}$ \\
\hline 5 & STU & Stair Up & 6 & $10 \mathrm{~s}$ \\
\hline 6 & WAL & Walking & 1 & $5 \mathrm{~m}$ \\
\hline
\end{tabular}

Accelerometer consists of three axes $\mathrm{X}, \mathrm{Y}$ and $\mathrm{Z}$. $\mathrm{X}$ and $\mathrm{Y}$ axis used for horizontal and vertical movements respectively. Z-axis is the third axis that measure data when body is in up and down position. For standing and sitting position, there is no gradual change in the 3 -axis measurements. When body is in walking activity, $\mathrm{X}, \mathrm{Y}$ and $\mathrm{Z}$ measurements are entirely different.

Table I shows each activities code, label, number of trails and duration taken for collecting data. Walking and standing activities takes 5 minutes duration for one trail. Dataset consist of timestamp, accelerometer $\mathrm{X}, \mathrm{Y}$ and Z-axis data respectively.

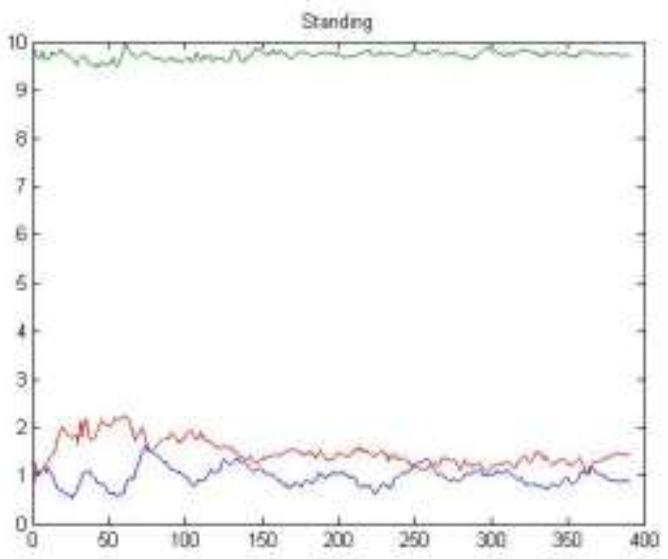

Fig. 3. Standing activity from Mobifall dataset

Fig. 3 shows an activity standing signal, reading from accelerometer. Dataset consist of 6 activities of daily livings (ADLs) such as jumping, sitting, standing, stair up, stair down and walking. Waking and standing data is taken with 5 minutes duration. All activities have a particular duration. In Fig $\operatorname{lref}\{$ four $\}$ consist of three signal shows $\mathrm{X}, \mathrm{Y}$ and $\mathrm{Z}$ axis data of accelerometer. Length of the signal is 400 .

\section{B. Data Preprocessing}

Data preprocessing is a technique which is used to transform the raw data in a useful and efficient format. May the data acquired from different sources consist of noise, unnecessary data, etc. This type of unwanted data should be removed. After data acquisition from the dataset, data undergoes data preprocessing. The processes included in data preprocessing are:

\section{- Data Cleaning}

In data cleaning, removing unnecessary information that are unwanted. The first few lines of each activity is regarding the activity that was removed. Noisy data or meaningless data are also removed.

\section{- Data Transformation}

Labelling process is mainly performed in the data transformation. Label each activity with their ids. For 


\section{International Journal of Engineering Applied Sciences and Technology, 2019 \\ Vol. 4, Issue 3, ISSN No. 2455-2143, Pages 374-381 \\ Published Online July 2019 in IJEAST (http://www.ijeast.com)}

jumping-label 1, sitting-label 2, standing-label 3, stair down-label 4, stair up-label 5 and walking-label 6.

\section{Feature Selection}

For human activity recognition, both time-domain features and frequency-domain features are considered. Time-domain features are feature related to signal amplitude with time. Features included in Time-domain features like mean, standard deviation, variance, etc. Features of Frequencydomain are signal features over the entire period. Frequencydomain features like signal entropy, spectral energy, kurtosis etc. Both features are listed in the Table 2.

TABLE 2. TIME AND FREQUENCY DOMAIN FEATURES

\begin{tabular}{|l|l|}
\hline Time Domain features & $\begin{array}{l}\text { Mean, standard deviation, Signal } \\
\text { magnitude area, variance signal } \\
\text { magnitude vector, skewness, tilt } \\
\text { angle. }\end{array}$ \\
\hline Frequency Domain Features & $\begin{array}{l}\text { signal entropy, spectral energy, } \\
\text { kurtosis }\end{array}$ \\
\hline
\end{tabular}

Mean, variance and standard deviation can be calculated directly from the data. For calculating a signal's mean use equation (1).

$$
\mu=\frac{1}{N} \sum_{i=0}^{N-1} x_{\mathrm{i}}
$$

RMS (Root Mean Square) value is useful for identifying walking pattern in human activity recognition. For calculating RMS value using equation (2)

$$
\text { RMS }_{\mathrm{X} \text {-axis }}=\sqrt{\frac{\sum_{i=1}^{n} x_{i}^{2}}{n}}
$$

Signal Magnitude Area (SMA) is used to distinguish between rest in order to identify when the subject is mobilizing and undertaking activities and periods of activity. SMA is implemented by equation (3) :

$$
\mathrm{SMA}=\frac{1}{i}\left(\sum_{u=1}^{i}\left|x_{u}\right|+\sum_{u=1}^{i}\left|y_{u}\right|+\sum_{u=1}^{i}\left|z_{u}\right|\right)
$$

Correspondingly, Signal Vector Magnitude (SVM) is calculated by equation (4). SVM is the useful for fall detection and is the intensity movement degree.

$$
\mathrm{SVM}=\sqrt{x^{2}+y^{2}+z^{2}}
$$

Tilt Angle(TA) is obtained by:

$$
T A=\arcsin \left(\frac{y_{i}}{\sqrt{x^{2}+y^{2}+z^{2}}}\right)
$$

One additional metric that distinguishes activity of daily living is energy. Energy can be calculated by:

$$
E_{\text {nergy }} y_{x-a x i s}=\frac{\sum_{i=1}^{\mathcal{W}} F F T_{x_{i}^{2}}}{n}-\mu
$$

Where FFT is another feature metric that can be calculated for each axis.

\section{Dimensionality Reduction}

After feature selection, dimension reduction is performed. That is transforming the original feature into a reduced feature set. For the dimension reduction, Principal component Analysis (PCA) and independent component Analysis (ICA) are used. First, PCA gives features that show highest variance. That features shows greatest variance called principal components. PCA algorithm is:

- Subtract mean vector from the original data

- $\quad$ Find co-variance matrix

- Calculate Eigen values and Eigen vectors of covariance matrix

- $\quad$ Find the principal components

- $\quad$ Derive the new dataset

Independent Component Analysis (ICA) finds each independent features in the data. ICA uses singular value decomposition here which is good as compared with PCA.

\section{E. Classification}

Proposed system uses two machine learning tools for classifying human activities. They are Multi class SVM and Naive Bayes. Multi class SVM is a new introduction in the area of human activity recognition. In multi class SVM, current class is set as 1 and consider all others as 0 . Then normal SVM classification procedure is done. SVM is used for Binary classification, But for more than two classes Multi class SVM is used.

\section{Support Vector Machine}

A machine learning approach is Support Vector Machine. Mainly SVM is used for classification. It can also be used for regression challenges. In classification, SVM is used to classify two classes called binary SVM. If SVM used for classify more than two classes then it is called multi class 
SVM. SVM takes number of features, and plot it into an ndimensional space. It performs classification by finding hyper plane that differentiate two classes.

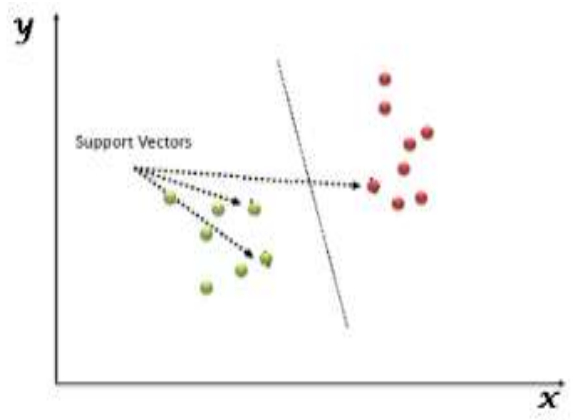

Fig. 4. Sample example of Support vector Machine

Vectors that define hyper plane is the support vector. Hyperplane should maximize the margin between two classes. In SVM, it is easy to have a linear separation between two classes. SVM has a technique called kernel trick. This function transforms lower dimensional data into a higher dimensional data. That is it transforms non-separable data into separable data. That is why this function called kernels. SVM is an example of supervised machine learning method.

\section{Multi class SVM}

Multi class SVM generalizes binary SVM. Multi class classification is an input is belongs to $\mathrm{K}$ classes. Here, the training data is the input data associated with class label, number from 1 to $\mathrm{K}$. When given a new data multi class SVM predict the class label. For example, in human activity recognition (HAR) to classify human activities.

\section{Naive Bayes}

Naive Bayes is a classification technique, which is based on Bayes' Theorem. Naive Bayes classifier supposed to be the feature in a class is unrelated to the presence of other feature. For example, recognizing human activities such as jumping, standing etc based on features mean, deviation, kurtosis, etc. Even if these features rely on each other or upon the fact of the other features, all of these features independently contribute to differentiate activities. So, this is called Naive. It is easy to build Naive Bayes model and useful for very large data sets particularly. By Bayes theorem, calculating posterior probability $\mathrm{P}(\mathrm{c} \mid \mathrm{x})$ from $\mathrm{P}(\mathrm{c}), \mathrm{P}(\mathrm{x})$ and $\mathrm{P}(\mathrm{x} \mid \mathrm{c})$. In the equation below:

$$
P(C \mid X)=\frac{P(X \mid C) P(C)}{P(X)}
$$

Where:

The posterior probability of class is $\mathrm{P}(\mathrm{c} \mid \mathrm{x})$.

The class prior probability is $\mathrm{P}(\mathrm{c})$.

The probability of predictor class is $\mathrm{P}(\mathrm{x} \mid \mathrm{c})$.
The predictor prior probability is $\mathrm{P}(\mathrm{x})$.

\section{EXPERIMENTAL EVALUATION}

Fig. 5 represents PCA as the dimensionality reduction. Figure. 5 shows the important principal components, where the first one shows a principal component variations from the total data which contains useful information.
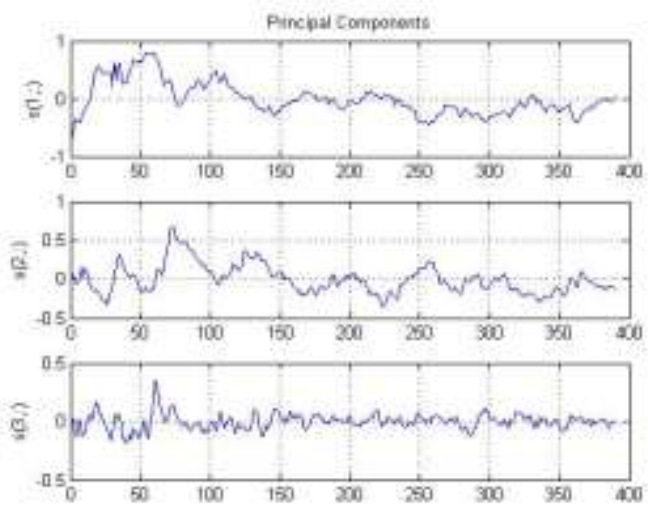

Fig. 5. Results after performing PCA

The next step is classification of the activities and then a comparison with their performance with classifiers. The comparison is done using concerning Precision, Recall, F-score and Accuracy. The first one in comparison is between the features extracted from the original data and features based on PCA. Based on classification tools, namely Multi Class SVM and Naive Bayes, the dataset is divided into two sets, where $70 \%$ is used for training set and $30 \%$ for the testing set process. Here, the features are normalized into the range of $[0,1]$.

TABLE 3. COMPARISION BETWEEN PCA-BASED FEATURES AND ICA-BASED FEATURES

\begin{tabular}{|c|c|c|}
\hline $\begin{array}{c}\text { Dimensionality } \\
\text { Reduction } \\
\text { Methods }\end{array}$ & Naive Bayes & $\begin{array}{c}\text { Multi Class } \\
\text { SVM }\end{array}$ \\
\hline PCA & $85 \%$ & $95.51 \%$ \\
\hline ICA & $75 \%$ & $94.08 \%$ \\
\hline
\end{tabular}

Table 3 shows the results from the comparison of classification performance between PCA-based features and ICA-based features. The classification is done using 207 samples. From Table III shows that, Multi Class SVM gives the best results compared to Naive Bayes, with the individual mean accuracy of $95.51 \%$. On the other hand, Naive Bayes gives relatively the worst results, with an accuracy of $85 \%$. From this table, the results also show that with PCA- based features and Multi Class SVM and Naive Bayes shows highest accuracy as compared with ICA. This is explained by the fact that PCA has reduced the feature dimensionality and only the highest variances (PC1, PC2 and PC3) are chosen as the dataset for the classification process. Thus, to improve 


\section{International Journal of Engineering Applied Sciences and Technology, 2019 \\ Vol. 4, Issue 3, ISSN No. 2455-2143, Pages 374-381 \\ Published Online July 2019 in IJEAST (http://www.ijeast.com)}

classification rate and classification process, dimensionality reduction process using PCA facilitates.

Table 4 shows the confusion matrix using the classifier Multi Class SVM. Data after dimensionality reduction process (PCA-based features) is the input to the classifier. The confusion matrix is subjected to perform between six classes of activities, namely standing (STD), sitting (SIT), stairs up (STU), stairs down (STN), walking (WAL), and Jumping (JUM). In the table, row represents the actual class and the column represents the predicted class by the classifier. Value in the table shows the probability of recognized actual class.

TABLE 4. Human ACTIVITy RECOGNITION CONFUSION MATRIX

\begin{tabular}{|c|c|c|c|c|c|c|}
\hline & JUM & $\mathrm{SCH}$ & STD & STN & STU & WAL \\
\hline JUM & 27 & 0 & 0 & 0 & 0 & 0 \\
\hline $\mathrm{SCH}$ & 1 & 52 & 1 & 0 & 0 & 0 \\
\hline STD & 0 & 1 & 8 & 0 & 0 & 0 \\
\hline STN & 0 & 0 & 0 & 54 & 0 & 0 \\
\hline STU & 0 & 0 & 0 & 19 & 35 & 0 \\
\hline WAL & 0 & 0 & 0 & 0 & 0 & 9 \\
\hline
\end{tabular}

Each individual activities performance measures like precision, recall, F-score and accuracy are shown in the Table V.

True Positive (TP) is the model correctly predict all classes.

False Positive (FP) is the positive class is incorrectly predicted.

False Negative (FN) is the rate of negative class is correctly predicted.

True Negative (TN) is the model correctly predict the negative classes.

Precision is calculated by:

$$
\text { Precision }=\frac{T P}{T P+F P}
$$

If activity model with no false positive, then precision is 1 . If the false positive rate is low then precision became high. Overall precision of the proposed is 0.9166 . Recall is also called sensitivity the ratio of true positive rate to all observations in actual class. Recall is find out from the equation (9)

$$
\text { Recall }=\frac{1 P}{\mathrm{TP}+\mathrm{FN}}
$$

Recall is good if it is above 0.5 . In the proposed system, an overall recall is 0.9289 . F-score or F-measure is obtained from equation (10).

$$
F-\text { score }=2 * \frac{\text { Precision } * \text { Recall }}{\text { Precision }+ \text { Recall }}
$$

Best value of F-score is 1 means that good precision and recall. The worst F-score obtained when low precision and recall, at a value of 0 . Overall F-score of the system is 0.9229 .

Finally, Accuracy is measured by:

$$
\text { Accuracy }=\frac{T P+T N}{T P+F P+F N+T N}
$$

Accuracy is an important metric in all classification-based system. Accuracy means how the proposed system or model accurate. Accuracy is the ratio of correct predictions to total number of samples. The proposed system gives an average accuracy $95.52 \%$.

TABLE 5. INDIVIDUAL ACTIVITY PERFORMANCE

\begin{tabular}{|c|c|c|c|c|}
\hline Activities & Precision & Recall & F-score & Accuracy \\
\hline JUM & 1 & 0.9642 & 0.9818 & $99.51 \%$ \\
\hline SCH & 0.9629 & 0.9811 & 0.9719 & $98.06 \%$ \\
\hline STD & 0.8889 & 0.8889 & 0.8889 & $98.99 \%$ \\
\hline STN & 1 & 0.7397 & 0.9503 & $87.58 \%$ \\
\hline STU & 0.6481 & 1 & 0.7865 & $88.95 \%$ \\
\hline WAL & 1 & 1 & 1 & $100 \%$ \\
\hline
\end{tabular}

\section{CONCLUSION}

In Human Activity Recognition, classification of ADLs based on a publicly available accelerometer dataset. The dataset uses an accelerometer sensor, which is embedded in a smartphone. The features based on time-domain and frequency-domain is selected from the original accelerometer signal dataset. PCA is performed on the original features to find the highest variance and reduction of the dimensionality of features. Evaluation of the approach by comparing the performance metrics such as precision, recall, F-score and accuracy of two types of machine learning classifiers. Individual activities performance is also measured.

A new probabilistic method for activity recognition can be used and compare the results with this system. Also, can try another type of activities like shopping, having dinner etc.

\section{ACKNOWLEDGMENTS}

The author would like to thank other authors' helps for preparing the paper. The dataset is publicly available [14]. Also like to thank each and every one for their contribution. 


\section{International Journal of Engineering Applied Sciences and Technology, 2019 \\ Vol. 4, Issue 3, ISSN No. 2455-2143, Pages 374-381 \\ Published Online July 2019 in IJEAST (http://www.ijeast.com)}

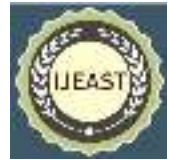

\section{REFERENCES}

[1] F. Attal, S. Mohammed, M. Dedabrishvili, F. Chamroukhi, L. Oukhellou, and Y. Amirat, "Physical Human Activity Recognition Using Wearable Sensors," Sensors, vol. 15, no. 12, pp. 31314-31338, 2015.

[2] Sztyler, "On body Localization of Wearable Devices : An Investigation of Position Aware Activity Recognition," 2016.

[3] C. Diniz ,D. Figo, P., D. R. Ferreira, and J. M. P. Cardoso, "Preprocessing Techniques for Context Recognition from Accelerometer Data," Pervasive Ubiquitous Computing., vol. 14, no. 7, pp. 645-662, 2010.

[4] C. Liu, Y. Hsu, and L. Fu, "Recognizing Context -aware Activities of Daily Living using RGBD Sensor," Iros2013, pp. 2222-2227, 2013.

[5] D. Chakraborty , Z. Yan, A. Misra, H. Jeung, and K. Aberer, "SAMMPLE: Detecting semantic indoor activities in practical settings using locomotive signatures," in Proc. of International Symposium on Wearable Computers, 2012.

[6] Y. Peng, L. Liu, M. Liu, and Z. Huang, "Sensor-based human activity recognition system with a multilayered model using time series shapelets," Knowledge-Based Systems, pp. 138-152, 2015.

[7] M. Rossi, J. Seiter, G. Tröster and O. Amft,, "Discovery of activity composites using topic models: An analysis of unsupervised methods," Pervasive and Mobile Computing, vol. 15, pp , 2014.

[8] S. Intille and L. Bao, "Activity recognition from user annotated acceleration data," in Proc. of International Conference on Pervasive Computing, 2004.

[9] V. Subbaraju, Z. Yan, D. Chakraborty, A. Misra, and K. Aberer, "Energy efficient continuous activity recognition on mobile phones: An activity adaptive approach," in Proc. of International Symposium on Wearable Computers, 2012.

[10] Q. Ji and Z. Zeng, "Knowledge Based Activity Recognition with Dynamic Bayesian Network," Network, 2010.

[11] S. Cho and Y. Lee, "Human Activity Inference Using Hierarchical Bayesian Network in Mobile Contexts", 2011.

[12] A. Patricio ,R. Cilla, M., J., J. M. Molina and García, A. Berlanga, "Recognizing Human Activities from Sensors using Hidden Markov Models Constructed by Feature Selection Techniques," Algorithms, 2009

[13] A. Zakaria , A.S Abdull Sukor, and N. Abdul Rahim, Activity Recognition using Accelerometer Sensor and Machine Learning Classifiers 978-1-5386-03895/18/31.00, 2018.
[14] M. Pediaditis, , M.Tsiknakis G.Vavoulas and E. G. Spanakis, The MobiFall dataset: An Initial Evaluation of Fall Detection Algorithms using Smartphones,13thIEEE Int. Conf. Bioinforma. Bioeng., no. November 2013.

[15] Osvaldo Simeone, "A Very Brief Introduction to Machine Learning With Applications to Communication Systems," IEEE Transl. J. Magn. Japan, vol. 4, pp. 740-741. December 2018

[16] Q. Wang et al., "Supervised and semi-supervised deep neural networks for CSI-based authentication," ArXiv eprints, Jul. 2018

[17] E. Sriperumbudur, “An Unsupervised Pattern Clustering Approach for Identifying Abnormal User Behaviors in Smart Homes,” Int. J. Comput. Sci. Netw, 2013.

[18] F. Jakab and M. Novák, "Approaches to a Problem of Anomaly Detection in User Behaviour in a Smart Home Environment," in Proceedings of the Faculty of Electrical Enginering and Informatics of the Technical University of Kosice, 2011.

[19] Y. Yoo and S. Bourobou "User Activity Recognition in Smart Homes Using Pattern Clustering Applied to Temporal ANN Algorithm," Sensors, 2015.

[20] C. Langensiepen, S. Mahmoud and A. Lotfi, "Behavioural Pattern Identification and Prediction in Intelligent Environments," Appl. Soft Comput. J, 2013.

[21] A. Arbor, C. L. R. Mcghan, A. Nasir, E. M. Atkins and A. Aerospace, "Human Intent Prediction Using Markov Decision Processes", 2012.

[22] , M. Fahim , I. Fatima, Y. K. Lee, and S. Lee, "A Unified Framework for Activity Recognition-based Behavior Analysis and Action Prediction in Smart Homes, 2013.

[23] W. Xiao and Y. Lu, "Daily Human Physical Activity Recognition Based on Kernel Discriminant Analysis and Extreme Learning Machine", 2015.

[24] L. Chen, C. Nugent, and G. Okeyo, "An Ontology-based Hybrid Approach to Activity Modeling for Smart Homes," IEEE Trans. Human-Machine Syst., 2014

[25] A. Gupta, P. Srinivasan, J. Shi, and L. Davis, "Understanding Videos, Constructing Plots-Learning a Visually Grounded Storyline Model from Annotated Videos," Proc. IEEE Conf. Computer Vision and Pattern Recognition, 2009.

[26] R. Jain and M. Shah, Motion-Based Recognition. Kluwer Academic, 1997.

[27] A. Hilton, T.B. Moeslund, , and V. Kruger, "A Survey of Advances in Vision-Based Human Motion Capture and Analysis," Computer Vision and Image Understanding, vol. 2, pp. 90-126, 2006. 